\title{
Resolving multiple sources of solar relativistic particles
}

\author{
Leon Kocharov ${ }^{* a}$, Andreas Klassen ${ }^{b}$, Ilya Usoskin ${ }^{a c}$ and Eino Valtonen ${ }^{d}$ \\ ${ }^{a}$ Sodankylä Geophysical Observatory (Oulu Unit), University of Oulu \\ P.O.B. 3000, FI-90014 Oulu, Finland \\ ${ }^{b}$ Institut für Experimentelle und Angewandte Physik, Christian-Albrechts-Universität \\ D-24118 Kiel, Germany \\ ${ }^{c}$ Department of Physics, University of Oulu \\ FI-90014 Oulu, Finland \\ ${ }^{d}$ Department of Physics and Astronomy, University of Turku \\ FI-20014 Turku, Finland \\ E-mail: leon.kocharov@oulu.fi,klassen@physik.uni-kiel.de, \\ ilya.usoskin@oulu.fi,eino.valtonen@utu.fi
}

\begin{abstract}
We consider the time-profile morphology of solar high-energy particle emissions, including relativistic electrons in three energy channels of $\mathrm{SOHO/EPHIN}$, relativistic protons as registered by the worldwide network of neutron monitors, and $\sim 100 \mathrm{MeV} / \mathrm{n}$ protons and helium in several energy channels of SOHO/ERNE. Based on numerical modeling of the interplanetary transport, we formulate a simple method for investigation of the high-energy particle sources operating at / near the Sun during the first hour of particle event. The method is applied to Ground Level Enhancement (GLE) and Solar Energetic Particle (SEP) events of the solar cycle 23. We conclude that depending on the GLE-SEP event scenario and detector vantage point, the observed particles originate from at least three sources. Possible nature of the sources is discussed in the framework of previous and new models of the high-energy particle production associated with global coronal (EIT) waves and CME shocks within about five solar radii from the Sun.
\end{abstract}

The 34th International Cosmic Ray Conference,

30 July- 6 August, 2015

The Hague, The Netherlands

\footnotetext{
* Speaker.
} 


\section{Method and results}

We start with modeling of the SEP transport from a source near the Sun to a detector at 1 AU. Interplanetary propagation of high-energy particles is modeled in the framework of focused transport [1] for the Kolmogorov turbulence spectrum in the standard solar wind. The energy dependence of the proton mean free path is according to the standard quasi-linear theory, while the mean free path of $\approx 1 \mathrm{MeV}$ electrons is equal to that of the $1 \mathrm{MeV}$ protons [2]. For analysis of real events, we simulate particle transport and registration with actual energy spectra, energy channels, aperture and viewing direction of a particular instrument. At large values of the mean free path, $\lambda \gtrsim 3 \mathrm{AU}$, the particle source profile may be estimated by a proper shifting of the instrument's counting rate profile back in time. The mean free path value is estimated using the flux anisotropy measurements.

Based on the results of particle transport and registration modeling, we shift the observed time-intensity profiles in time back to the Sun but add eight minutes to facilitate a comparison with electromagnetic emissions observed at $1 \mathrm{AU}: t_{\mathrm{S}}=t-\Delta t+8 \mathrm{~min}$. The time-shifted and renormalised profiles of the 13 December 2006 event (GLE 70) are shown in Figure 1. Plotted only the neutron monitor profile revealing the earliest and steepest onset in this GLE. For a convenience of comparison of different particle species and energy channels, the background levels are reduced to one and the same level that is $1 \%$ of the real neutron monitor background.

\section{Discussion and conclusions}

Inspection of $\sim(0.1-1) \mathrm{GeV} / \mathrm{n}$ protons and helium and $\sim(0.3-3) \mathrm{MeV}$ electrons of GLEs associated with flares at $\sim\left(10^{\circ}-25^{\circ}\right) \mathrm{W}$ has revealed at least three particle sources: Source 1 is a short source associated with flare and CME launch (Period 1 in Figure 1); Source 2 is a prolonged source operating from Period 1 through Period 3, naturally identified with the CME bow shock on open magnetic field lines extending to the solar wind; and Source 3 releases particles in Period 2, could be a compact structure left behind the shock. Source 3 is responsible in particular for the prompt component of GLE. Delayed component of the GLE may contain helium recycled from Source 3.

Different sources, however, do not necessarily suggest different acceleration mechanisms. For instance, a shock can produce different energy spectra and time profiles depending on the environment met, which may include different magnetic structures, plasma turbulence and seed particle populations [3, 4]. Possible scenario is illustrated with Figure 2.

\section{Acknowledgments}

We thank the $A C E /$ MAG instrument team, the $A C E$ Science Center, NOAA and IZMIRAN for providing the data of $A C E, G O E S$, and neutron monitors. Oulu neutron monitor data are available at http://cosmicrays.oulu.fi. We use the CME catalog generated and maintained at the CDAW Data Center by NASA and The Catholic University of America in cooperation with the Naval Research Laboratory. $\mathrm{SOHO}$ is a project of international cooperation between ESA and NASA. This research was supported by the Academy of Finland through project 260596. 


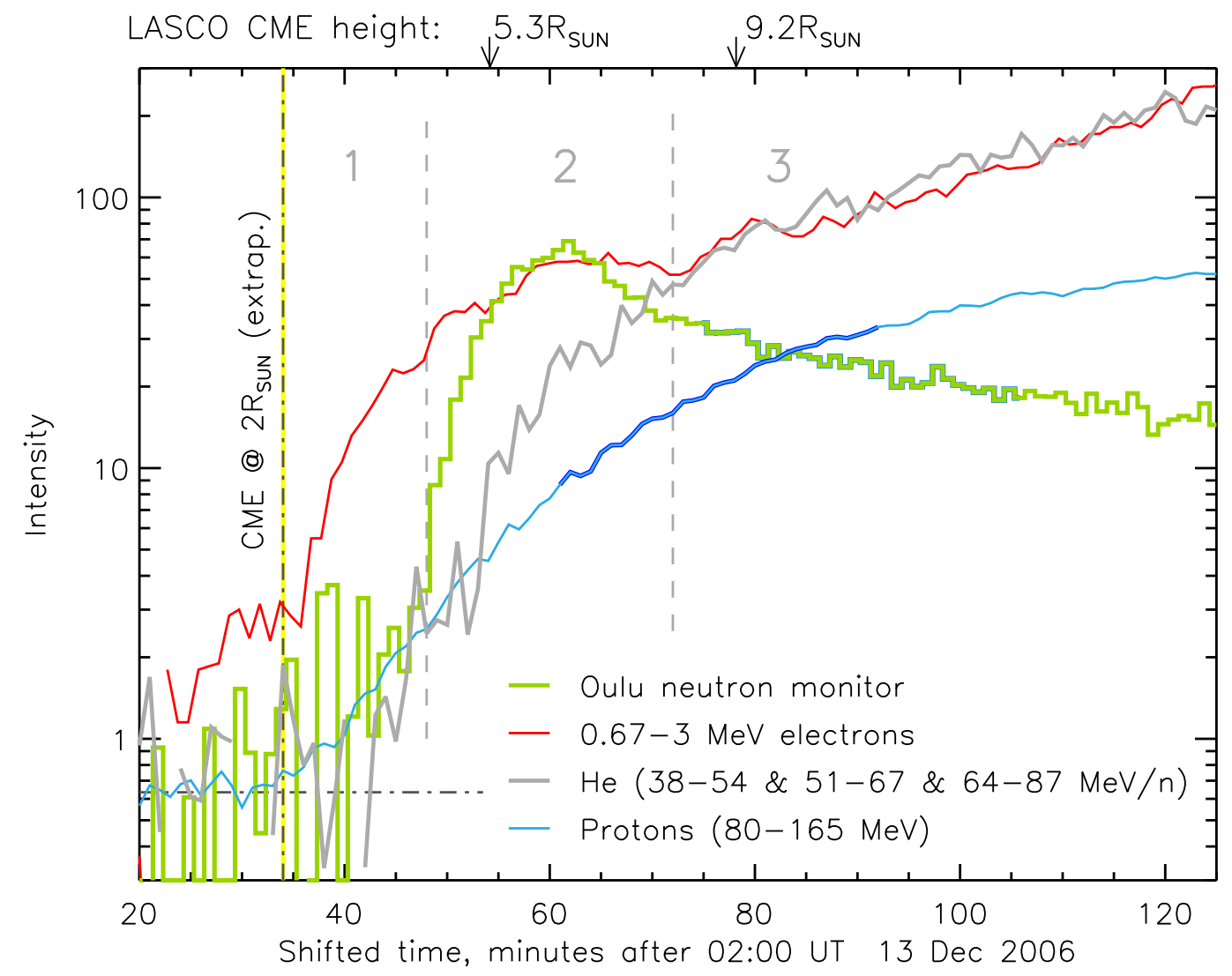

Figure 1: Time-shifted profiles of the 13 December 2006 SEP and GLE event (GLE 70). Shown are the time-shifted and re-normalized profiles of the Oulu neutron monitor count rate (shifted by $-3 \mathrm{~min}$ ), the proton channel P6 (80-165 MeV) of GOES 11 (-17 min), the 0.67-3 MeV electrons from SOHO/EPHIN ( $-4 \mathrm{~min})$, and the sum of three time-shifted profiles of helium (SOHO/ERNE). The helium channels (and time shifts) are 38-54 MeV/n (-28 min), 51-67 MeV/n (-24 min), and 64-87 MeV/n (-20 min). Most of the neutron monitor background is subtracted (99\%). All other background levels are reduced to the remnant background of the neutron monitor. The He/p abundance ratio in the 64-87 MeV/n channel of ERNE in early phase of the event, $t_{\mathrm{S} 1}=46-53 \mathrm{~min}$, is $(\mathrm{He} / \mathrm{p})_{1}=0.95 \pm 0.25 \%$. Simultaneously with the rise of the GLE's prompt component (in Period 2), the helium abundance increases by a factor of $2.8 \pm 0.5$ (between the $t_{\mathrm{S} 1}$ and $t_{\mathrm{S} 2}=57-74 \mathrm{~min}$ ). For a comparison with PAMELA data [5], we indicate the shifted time interval that corresponds to the first period of PAMELA observations, 03:18-03:49 UT (thicken segments of the neutron monitor and GOES profiles). At the high-energy end, PAMELA observed only the delayed component of GLE 70 (Period 3), while on the whole, the observed spectra are the weighted sum of ions from at least two different sources. 

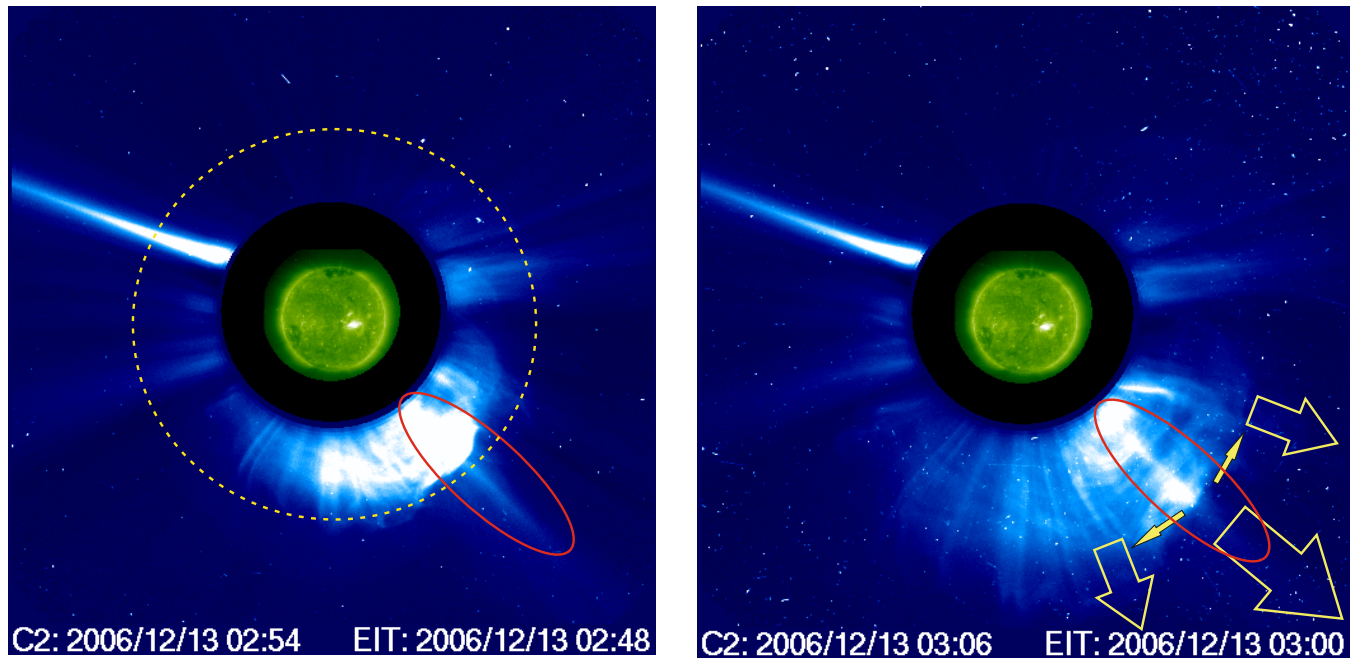

Figure 2: Hypothetical source region of GLE 70. Left: SOHO LASCO and EIT images of solar corona at the main rise of the GLE's prompt component (the rise started at $t_{\mathrm{S}}=02: 48 \mathrm{UT}$, Figure 1). Yellow circle shows the height of the LASCO CME at 02:48 UT (quadratic extrapolation). Red oval shows location of a previous brightening at intersection of the streamer with preceding ejection, which was observed within half an hour prior to the GLE. Right: Red oval is possible acceleration and/or release region of the GLE's prompt (core) component. A pair of tangential arrows illustrates the cross-field transport of accelerated particles, which may populate the CME's loops and contribute to the delayed (halo) component of GLE. Alternatively, the delayed component may be produced far aside of the core at the lateral expansion associated with CME liftoff and visualised close to the Sun by the EUV wave. Radial arrows illustrate the particle escape to interplanetary medium.

\section{References}

[1] L. Kocharov, V.J. Pizzo, D. Odstrcil, and R.D. Zwickl, A unified model of solar energetic particle transport in structured solar wind, JGR 114 (2009) A05102

[2] J.W. Bieber, W.H. Matthaeus, C.W. Smith, W. Wanner, M.-B. Kallenrode, and G. Wibberenz, Proton and electron mean free path: The Palmer consensus revised, ApJ 420 (1994) 294

[3] A. Afanasiev, R. Vainio, and L. Kocharov, The effect of stochastic re-acceleration on the energy spectrum of shock accelerated protons, ApJ 790 (2014) 36

[4] L. Kocharov, T. Laitinen, R. Vainio, A. Afanasiev, K. Mursula, and J.M. Ryan, Solar interacting protons versus interplanetary protons in the core plus halo model of diffusive shock acceleration and stochastic re-acceleration, ApJ 806 (2015) 80

[5] O. Adriani, O. et al., Observations of the 2006 December 13 and 14 solar particle event in the 80 $M e V n^{-1}-3 \mathrm{GeV} n^{-1}$ range from space with the PAMELA detector, ApJ 742 (2011) 102 